\title{
Topoisomerases and yeast rRNA transcription: negative supercoiling stimulates initiation and topoisomerase activity is required for elongation
}

\author{
Michael C. Schultz, ${ }^{1}$ Steven J. Brill, ${ }^{2,3}$ Qida Ju, ${ }^{4}$ Rolf Sternglanz, ${ }^{2}$ and Ronald H. Reeder ${ }^{1}$ \\ ${ }^{1}$ Basic Sciences Division, Hutchinson Cancer Research Center, Seattle, Washington 98104 USA; ${ }^{2}$ Department of \\ Biochemistry, State University of New York, Stony Brook, New York 11794 USA; ${ }^{4}$ Department of Cell Biology, Albert \\ Einstein College of Medicine, Bronx, New York 10461 USA
}

\begin{abstract}
Previous work has shown that rRNA synthesis is strongly inhibited in yeast top1-top2 double mutants. Here, we show that inactivation of yeast topoisomerases can have paradoxical effects on transcription by RNA polymerase I. For example, transcription of ribosomal minigenes on extrachromosomal plasmids is greatly stimulated in top1-top2 cells while accumulation of full-length endogenous rRNA is strongly inhibited. We present evidence for a mechanism that can partly account for these opposing effects on transcription. On the one hand, transcription initiation can be stimulated owing to an accumulation of negative superhelicity because polymerase I prefers to initiate on negatively supercoiled templates. Conversely, synthesis of full-length rRNA is inhibited owing to the fact that chain elongation requires a DNA relaxing activity.
\end{abstract}

[Key Words: Topoisomerases; yeast; RNA polymerase $I_{\text {; }}$ transcription; supercoiling]

Received December 6, 1991; revised version accepted April 20, 1992.

Accumulation of the large rRNAs in both budding and fission yeast requires the presence of topoisomerase activity in the cell (Brill et al. 1987; Yamagishi and Nomura 1988). The accepted explanation for this observation is that topoisomerase is required for efficient RNA chain elongation, and RNA polymerase I abortively terminates in its absence. Both theoretical considerations and some elegant in vivo experiments suggest that an elongating RNA polymerase complex causes an accumulation of positive supercoils in front of it and an accumulation of negative supercoils behind it (Liu and Wang 1987; Brill and Sternglanz 1988; Giaever and Wang 1988; Wu et al. 1988). This accumulation of superhelical stress can be relieved either by diffusion, if the DNA is free to rotate, or by topoisomerase action. If neither relaxation process is available (or is too slow), the accumulation of positive supercoils ahead of the polymerase will eventually reach a point where elongation ceases. In yeast, either topoisomerase I or II can supply the relaxation activity for efficient rRNA transcription (Brill et al. 1987; Yamagishi and Nomura 1988). When both topoisomerases are inactivated, however, rRNA accumulation is severely inhibited.

Although the model just presented can explain many of the known facts concerning topoisomerase and tran-

${ }^{3}$ Present address: Cold Spring Harbor Laboratory, Cold Spring Harbor, New York 11724 USA. scription, some questions still remain. For example, the possibility remains that topoisomerases might be required for efficient initiation of transcription as well as for elongation. Initiation of transcription requires localized denaturation of the DNA with concomitant generation of positive superhelicity. It is possible, therefore, that frequent initiation, such as occurs at a ribosomal gene promoter, might be aided by topoisomerase. Until now there has been no direct evidence either for or against this possibility. An additional problem is that in some cases, inactivation of topoisomerase does not cause the expected inhibition of RNA polymerase I transcription. For example, Zhang et al. (1988) noted a slight stimulation of nuclear run-on transcription near the $5^{\prime}$ end of the human rRNA genes when topoisomerase I was inhibited with camptothecin. Likewise, Dunaway (1990) observed an increase in rRNA transcription when oocytes were treated with VM-26, an inhibitor of topoisomerase II. And, as we show in this paper, inactivation of topoisomerase can cause a dramatic increase in the transcription of some artificial polymerase I transcription units in yeast.

We present evidence for a model that explains how inactivation of topoisomerase can stimulate transcription in some cases while inhibiting it in others. Inactivation of topoisomerase actually has at least two effects. One effect has already been discussed, that is, the inhibitory effect caused by the buildup of positive superhelic- 
ity ahead of the elongating polymerase and the inability of polymerase to elongate in the absence of relaxing activity. The second effect is a corollary of the first effect and can result in a stimulation of transcription initiation. If positive superhelicity accumulates ahead of the polymerase, negative superhelicity can be expected to accumulate behind it (Liu and Wang 1987). In this paper we show that initiation by RNA polymerase I is strongly stimulated by negative superhelicity. Thus, in the absence of topoiosomerase activity, the net transcription of an RNA polymerase I transcription unit can be viewed as the sum of two opposing forces: a stimulation of initiation coupled with an inhibition of RNA chain elongation. This model can explain many, but not all, of the in vivo effects that topoisomerase inactivation has on polymerase I transcription.

\section{Results}

In the first part of this study, we have examined how the transcription of ribosomal genes in the intact yeast cell responds to loss of topoisomerase activity. Three variables have been altered. First, we have utilized several different ribosomal gene constructs, with and without the rDNA enhancer and of various overall length. These constructs are diagramed in Figure 1. Second, the location of these constructs within the cell has been varied by introducing them either as extrachromosomal plasmids or into various chromosomal locations. Finally, the transcription of these constructs has been assayed under various conditions of topoisomerase inactivation. The results of these assays are shown in Figures 2-4.

\section{rDNA constructs for the assay of $r R N A$ transcription} in vivo

The ribosomal genes of Saccharomyces cerevisiae are arranged as a tandem repeat of $\sim 200$ copies. To study the transcription of a single member of this gene family, we have utilized some constructs originally made by Elion and Warner (1984), in which a unique fragment of phage T7 DNA is inserted between the ribosomal gene promoter and 3 '-end-forming sites (Fig. 1A). Construct rR8 contains both the $5^{\prime}$ and $3^{\prime}$ ends of the gene but lacks the enhancer element. Construct rR10 is similar to rR8 but also contains the EcoRI-HindIII enhancer fragment in its normal location. $r R 8$ and $\mathrm{rR} 10$ are relatively short minigenes, producing transcripts of $1.2 \mathrm{~kb}$. To enlarge these minigenes to a size similar to that of the rRNA precursor coding unit we inserted an additional 4.9-kb fragment of phage $\lambda$ DNA immediately downstream of the T7 sequences, as shown in Figure 1A, to make the maxigenes rR6.1 (without the enhancer) and rR6.2 (with the enhancer). These four constructs were the starting point for the in vivo transcription experiments we now describe.

\section{Transcription from plasmid-borne constructs in toposiomerase mutants}

The ribosomal minigenes, $\mathrm{rR} 8$ and $\mathrm{rR} 10$, as well as the maxigene rR6.2, were introduced as extrachromosomal plasmids into a set of four yeast strains that were either wild type or mutant in one or both topoisomerases. As shown in Figure 2, all three of the constructs exhibit a similar response to topoisomerase mutation. Deletion of topoisomerase I causes a small but reproducible increase in transcription. Shifting the top2 mutant to the nonpermissive temperature causes a much larger increase in transcription. In the top1-top2 double mutant, all of the constructs show an increase in transcription over wild type, although the increase is not as great for the longer construct, rR6.2. In addition, short transcripts from rR6.2 accumulate in both the top2 and double mutant, suggestive of premature termination.

The consistent picture obtained with the extrachromosomal constructs is that inactivation of either topoisomerase causes an increase in rDNA transcription, and inactivating both topoisomerases causes an even larger increase in two of three cases. This effect is independent of the presence or absence of the enhancer element. These results caused some surprise. Previous studies of rRNA transcription from the endogenous ribosomal genes on chromosome XII showed that transcription was inhibited severely in the double mutant (Brill et al. 1987). The fact that we consistently observe an increase in transcription from extrachromosomal genes with the same double mutant suggests that some additional factors must be taken into account.

\section{Transcription from constructs integrated into the chromosome}

An obvious possibility is that ribosomal genes on extrachromosomal plasmids are under different topological constraints than genes situated in a chromosome, and this might account for the unexpected result seen in Figure 2. To examine this possibility, ribosomal gene constructs were integrated into two different chromosomal locations, either the URA3 locus or the rDNA locus, and their response to topoisomerase inactivation was assayed. Figure 1B shows the structure of the chromosomal integrants at both loci.

Figure 3 shows the expression levels from constructs integrated at the URA3 locus. For the minigene construct $\mathrm{rR10}$, the result is qualitatively similar to that seen with extrachromosomal plasmids. Inactivating either topoisomerase I or II causes an increase in the transcription signal, and an even larger increase is seen in the double mutant. For the maxigene rR6.1, a different result is obtained. While inactivating either topoisomerase I or II causes a stimulation of transcription, there is a severe inhibition of signal in the double mutant. These results can be interpreted to mean that on a short gene, rR10, results are similar whether the construct is integrated or extrachromosomal. However, on a longer gene, rR6.1, the integrated construct is more sensitive to topoisomerase inactivation than is the extrachromosomal construct, and severe inhibition of transcription is observed in the case of the double mutant.

Figure 4 illustrates that the same qualitative result is obtained when constructs are integrated into the rDNA 
Figure 1. Yeast rDNA constructions. Constructions with the control elements for transcription by RNA polymerase I were maintained in yeast as plasmids $(A)$ or as integrants into a chromosome $(B)$. All constructs were derived from rR8 (Elion and Warner 1984) and contained the 2.35kb HindIII-BglII fragment of the repeat (top diagram), which includes the $5 \mathrm{~S}$ gene and the polymerase I promoter; the 590 -bp EcoRI fragment, which includes the $3^{\prime}$ end of the coding region; and $600 \mathrm{bp}$ of $\mathrm{T} 7 \mathrm{ph}-$ age DNA (light hatched box). (A) The rR8 minigene differed from $\mathrm{rR} 10$ by the restoration of the 190-bp EcoRI-HindIII enhancer fragment to its normal location in rR10. The maxigenes (rR6.1 and rR6.2) both contained an additional $4.9 \mathrm{~kb}$ of phage $\lambda$ DNA (dark hatched box) inserted between the T7 DNA and the 590-bp fragment from the $3^{\prime}$ end of the ribosomal gene repeat. rR6.1 differed from rR6.2 by the restoration of the enhancer fragment, in rR6.2, to the 5' end of the large fragment from the repeat. $(B)$ The minigene and maxigene constructs in $A$ were integrated into the ribosomal gene repeat along with the $U R A 3$ gene $(1,3)$, or they were transplaced to the repeat such that the introduced constructs were surrounded only by rDNA sequences $(2,4)$. The minigene and maxigenes were also integrated into the $U R A 3$ locus of the appropriate host strain bosomal genes was assayed by probing for the T7 RNA on Northern blots. The minigenes and maxigenes produce 1.2- and 6.1$\mathrm{kb}$ transcripts, respectively. Restriction sites: (Bg) BglII; (E) EcoRI; (H) HindIII; (S) SmaI. $(5,6)$. Transcription from the artificial ri-
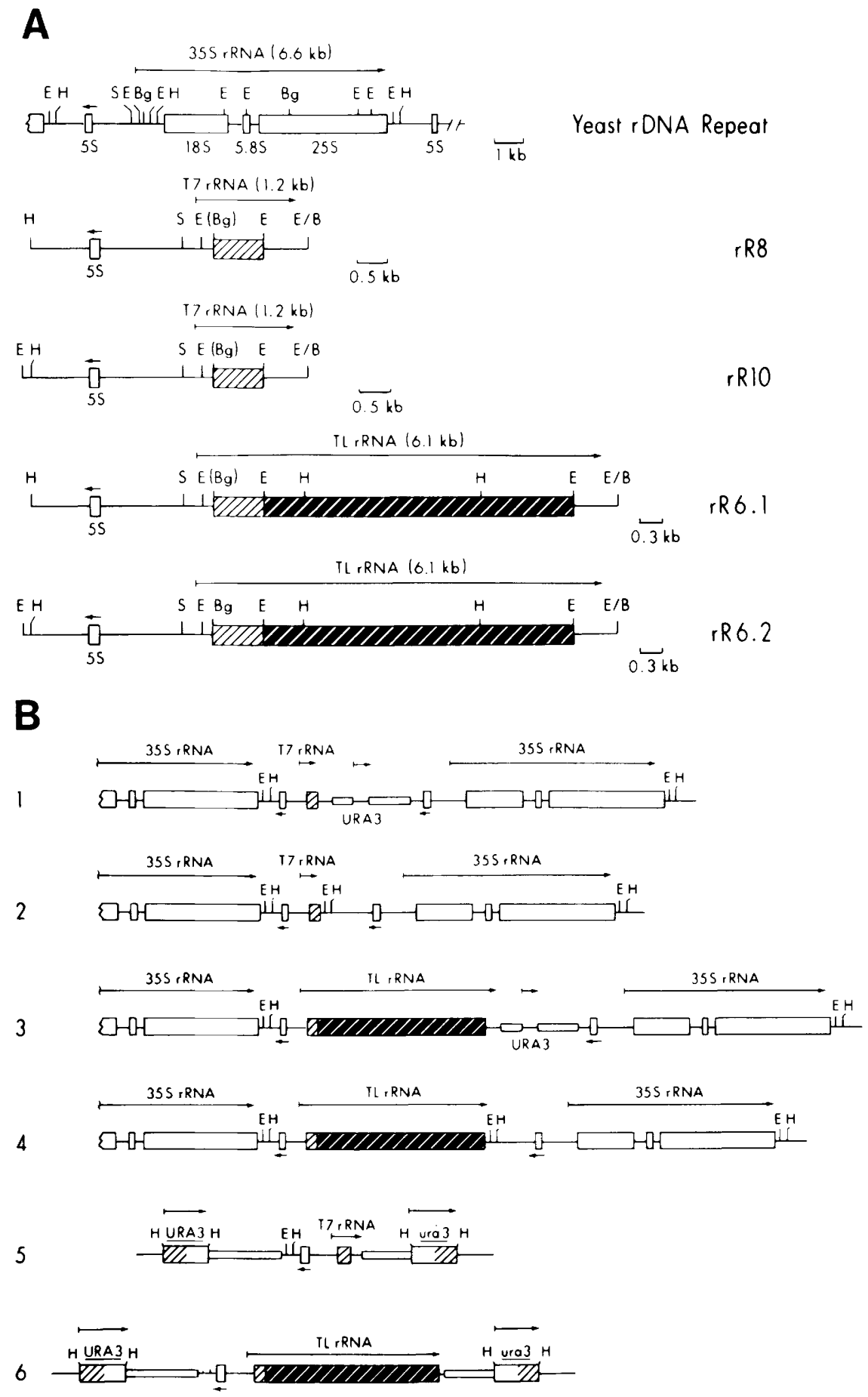

locus, whether or not the URA3 selectable marker and vector sequences are allowed to remain or are eliminated. In Figure 4, A and B, we see that inactivation of both topoisomerases causes a significant increase in transcription of the short construct, just as was observed when it was integrated into the URA3 locus (Fig. 3A) or when it was on an extrachromosomal plasmid (Fig. $2 \mathrm{~A}, \mathrm{~B})$. In contrast, transcription from the longer maxigene construct is inhibited severely upon inactivation of both topoisomerases (Fig. 4C,D). This is the same behavior that was observed when a maxigene construct was integrated at the URA3 locus (Fig. 3B).

\section{A hypothesis to account for the in vivo effects of topoisomerase inactivation upon ribosomal gene transcription}

If we combine the data for transcription of ribosomal 


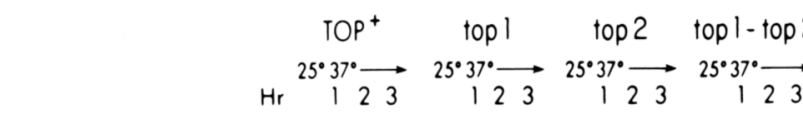

A. YCprR8
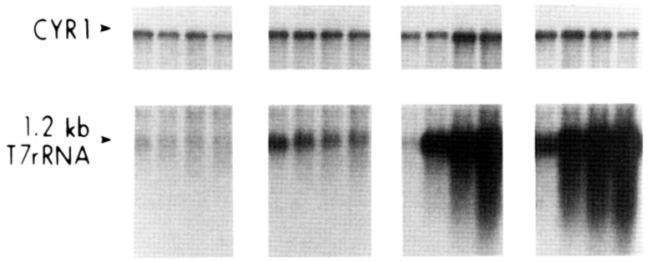

B. YCprRIO
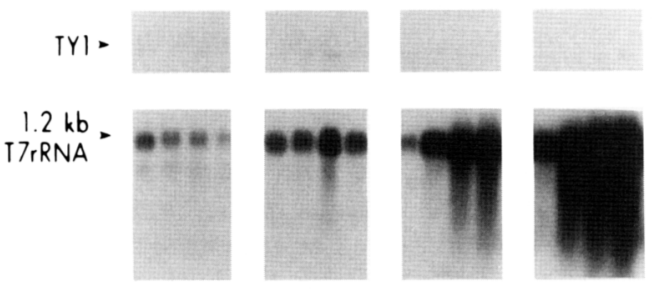

C. YCprR6.2
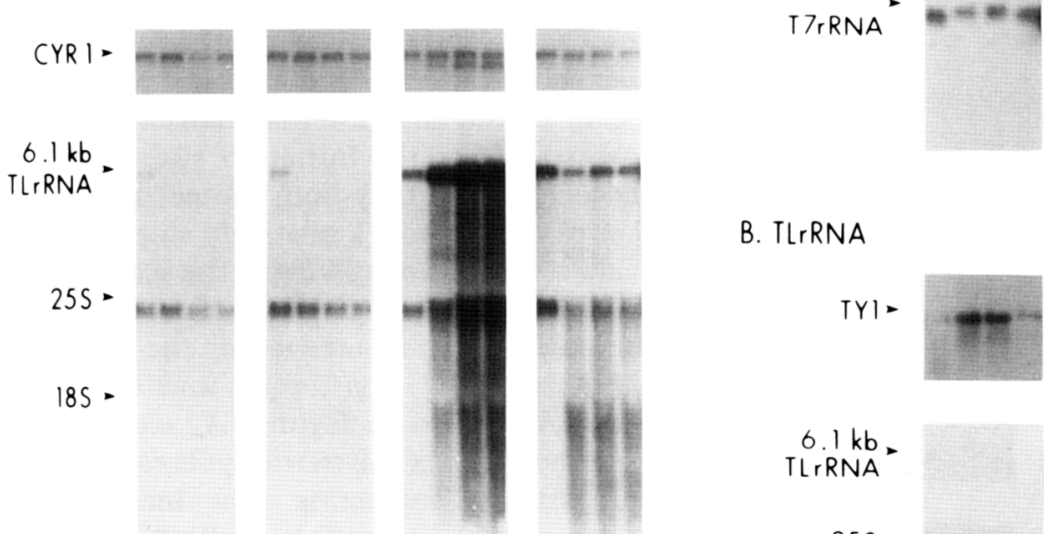

$1234 \quad 5678 \quad 9101112 \quad 13141516$

Figure 2. RNA polymerase I transcription levels on plasmid constructs. $\mathrm{TOP}^{+}$, top1, top2, and top1-top 2 cells carrying plasmids with the genes $\operatorname{rR} 8(A), \operatorname{rR} 10(B)$, or $\operatorname{rR6} 2(C)$ were grown in YEPD medium at $25^{\circ} \mathrm{C}$ and shifted to $37^{\circ} \mathrm{C}$. At the indicated times, total nucleic acids were isolated, and transcription from the artificial rDNA constructs (T7 and TL rRNA in Figs. 2-4) and an internal control (CYR1 or TY1 in Figs. 2-4) was analyzed by Northern blotting. In each case, the top1 mutant supports slightly higher levels of rDNA transcription than the wild type. Inactivating topoisomerase II by shifting the top 2 cells to $37^{\circ} \mathrm{C}$ greatly stimulates transcription. Cells with no topoisomerase I or topoisomerase II activity (top1-top 2 cells shifted to $37^{\circ} \mathrm{C}$ ) show heavy transcription from the $\operatorname{rR} 8(A)$ and $\operatorname{rR} 10(B)$ constructs.

mini- and maxigenes presented in Figures $2-4$ with results already published for the endogenous genes (Brill et al. 1987), we see that inactivation of topoisomerases can have different effects upon transcription by RNA polymerase I, depending on the size of the transcription unit and possibly on its plasmid or chromosomal location. In some cases, transcription is strongly stimulated /see Fig. 2A), and in others, it is strongly inhibited (see Fig. 4D). We can go some ways toward explaining these results if
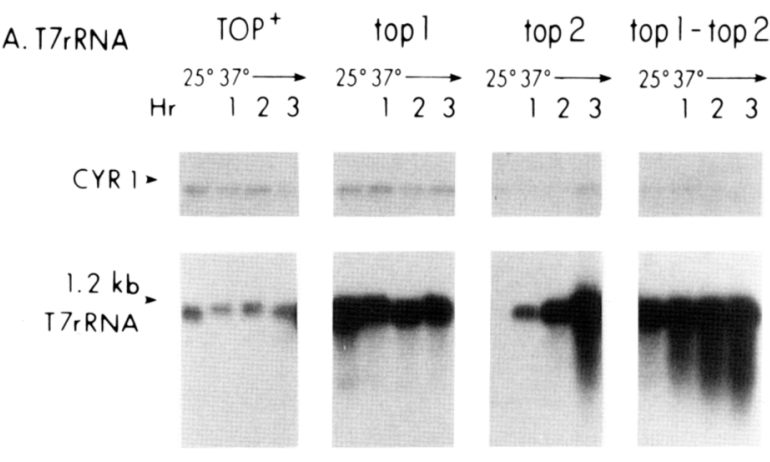

B. TLrRNA

we assume that inactivation of topoisomerases has two separate effects: one that stimulates rRNA transcription initiation, and one that inhibits transcription elongation In this view, the transcription signals measured in Fig cesses.

We propose the following hypothesis: As proposed pretranscription by inhibiting the chain elongation process. This effect is more severe on long genes than on short

B. TLRRA
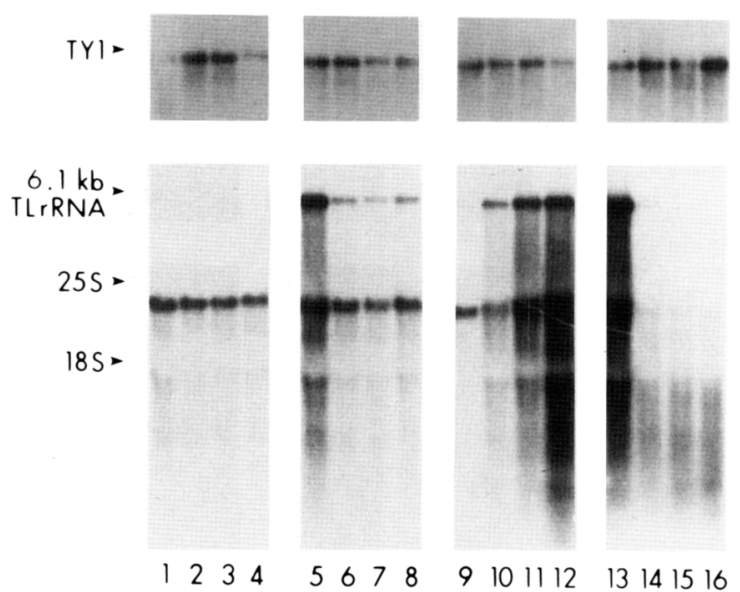

Figure 3. RNA polymerase I transcription levels on constructs integrated at the URA3 locus. (A) T7rRNA. The IR10 minigene was integrated at the URA3 locus of $T O P^{+}$, top1, top2, and top1-top2 cells. The resultant strains were grown at $25^{\circ} \mathrm{C}$ and shifted to $37^{\circ} \mathrm{C}$ for the indicated times. RNA was analyzed by Northern blotting by use of a probe against the T7 phage insert in the minigene. Transcription from the rR10 minigene is elevated in cells lacking either topoisomerase I (top1) or topoisomerase II (top2 at $37^{\circ} \mathrm{C}$ ). Cells with neither activity (top 1-top2 at $37^{\circ} \mathrm{C}$ ) show even higher transcription from rR $10 .(B) \mathrm{TL}$ rRNA. This is the same as $A$, except that the rR6.1 maxigene was integrated at the URA3 locus and RNA was analyzed by Northern blotting by use of a probe against the phage $\lambda$ DNA insert in the maxigene. Transcription from the rR6.1 maxigene is also elevated in cells with either topoisomerase I deleted (top1) or with topoisomerase II inactivated (top 2 at $37^{\circ} \mathrm{C}$ ). However, when both enzymes are inactivated by shifting top 1-top 2 cells to $37^{\circ} \mathrm{C}$, transcription from the maxigene is severely inhibited. 


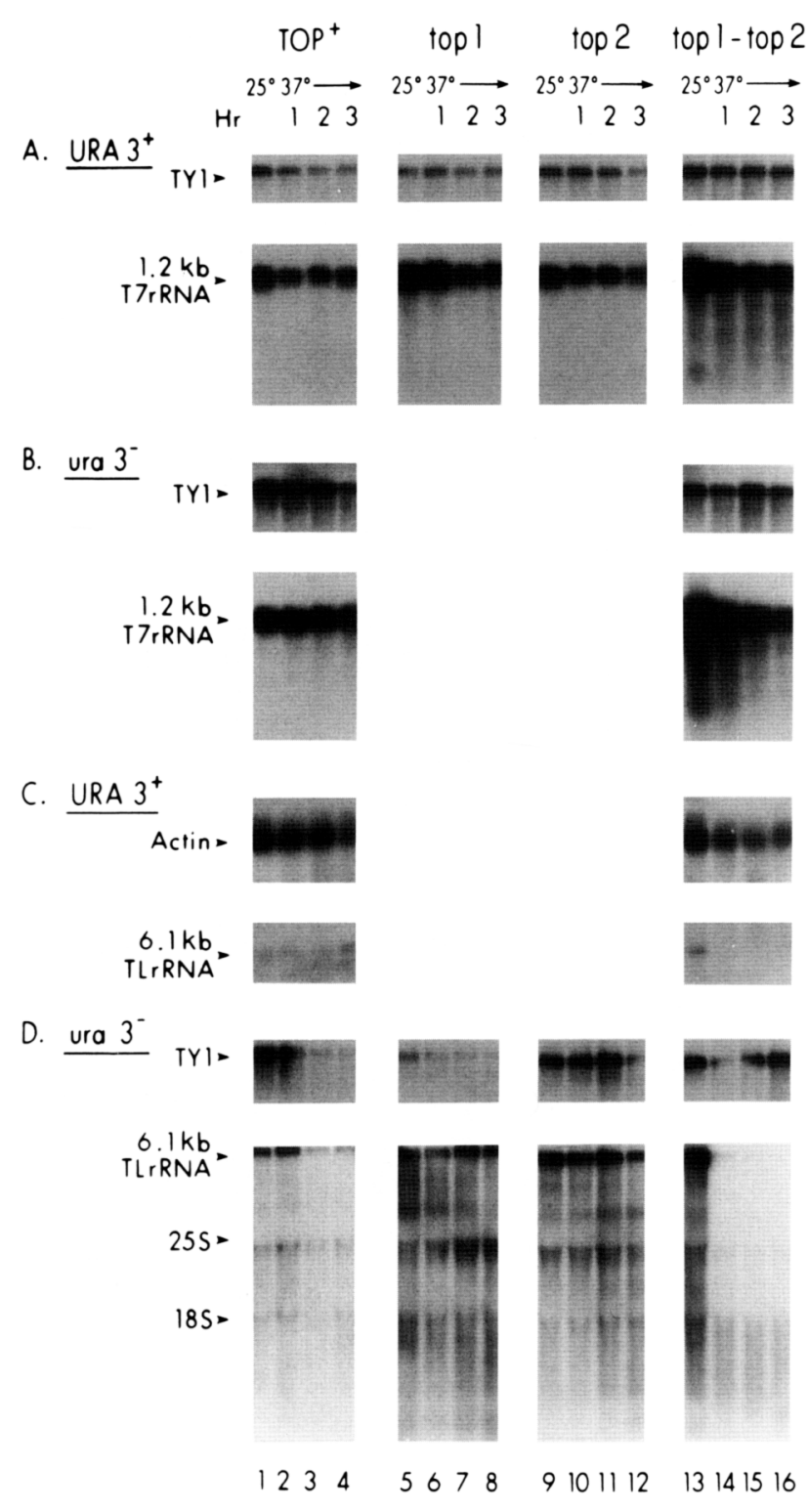

Figure 4. RNA polymerase I transcription levels on constructs integrated and transplaced at the rDNA locus. $(A) U R A 3^{+}$. The rR8 minigene was integrated at the rDNA locus along with the $U R A 3$ gene. The resultant strain was grown at $25^{\circ} \mathrm{C}$ and shifted to $37^{\circ} \mathrm{C}$ for the indicated times. RNA was analyzed by Northern blotting by use of a probe against the phage T7 sequence in the minigene. $(B)$ ura $3^{-}$. This is the same as $A$, except that the rR8 construct was transplaced so that it is surrounded only by rDNA. (C) $U R A 3^{+}$. This is the same as A, except that the rR6.1 maxigene construct was integrated at the rDNA locus and RNA was analyzed using a probe against the phage $\lambda$ sequence in the maxigene. $(D) u r a 3^{-}$. This is the same as $C$, except that the rR6.1 construct was transplaced so that it is surrounded only by rDNA. Note that transcription from the minigene rR8 is stimulated in the double mutant $(A, B)$. The opposite effect is observed for the maxigene: When both enzymes are inactivated by shifting top1-top 2 cells to $37^{\circ} \mathrm{C}$, transcription is severely inhibited $(C, D)$. genes and is also more severe on a chromosome than on an extrachromosomal plasmid. At the same time, inactivation of topoisomerases causes the accumulation of negative superhelicity, at least transiently. We propose further that RNA polymerase I initiates more readily on negatively supercoiled templates; thus, there is an opposing tendency to increase transcription when topoisomerase is inactivated. In the following text, we describe experiments that support this hypothesis.

\section{Levels of endogenous $r$ RNA transcription \\ in topoisomerase mutants as measured at different points in the transcription unit}

According to the hypothesis, inhibition of transcription in topoisomerase mutants is the result of an elongation defect and should therefore increase in severity as transcription is monitored at greater distances from the site of initiation. To test this prediction we made two endlabeled single-stranded DNA probes (Fig. 5): one that protects the extreme 5 ' end of the endogenous ribosomal precursor transcript, and a second probe that protects a region of the first internal transcribed spacer, $2.6 \mathrm{~kb}$ downstream of the initiation site. These probes were chosen because they both protect regions of the RNA that turn over with a relatively short half-life; thus, we avoid complications owing to the accumulation of stable RNA in the cytoplasm. The probes were used to measure the steady-state abundance of the relevant regions of rRNA in $\mathrm{TOP}^{+}$, top1, top2, and top1-top2 strains of yeast. As shown in Figure $5 \mathrm{~A}$, accumulation of RNA from the $5^{\prime}$ end of the transcript is essentially equal in all four strains, suggesting that inactivation of topoisomerases has little effect on RNA polymerase I initiation on the endogenous genes. A different result is obtained when the downstream probe is used (Fig. 5B). The single mutants are relatively unaffected, indicating that either topoisomerase is sufficient for chain elongation. However, in the double mutant, the downstream signal is severely impaired, suggesting that most polymerases abortively terminate before progressing $2.6 \mathrm{~kb}$ down the gene.

From this experiment we conclude that chain elongation is the major cause of transcription inhibition in topoisomerase mutants, and the magnitude of the defect will clearly be greater for long transcripts than for short transcripts. We also note that there was neither a stimulation nor an inhibition of initiation on the endogenous ribosomal genes.

\section{Initiation on a supercoiled template is stimulated in extracts from the top1-top2 strain}

Although RNA polymerase I apparently does not require topoisomerase for efficient initiation, initiation in vitro can be stimulated by the absence of topoisomerase. In comparing transcription extracts made from wild-type or top1-top2 mutant strains, we have consistently observed an $\sim 10$-fold higher initiation rate in the mutant extracts 


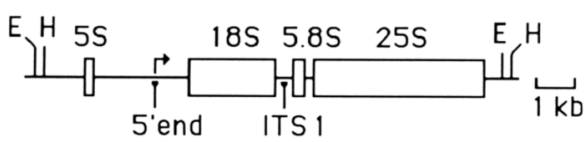

$A$

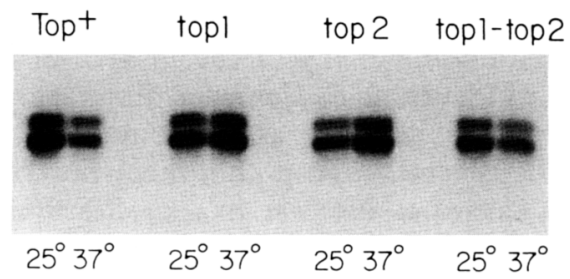

$B$

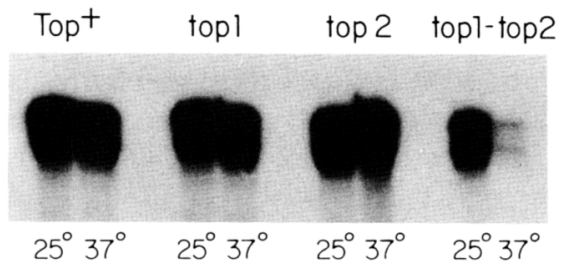

Figure 5. Levels of endogenous rRNA transcription in topoisomerase mutants. Endogenous ribosomal gene transcription was measured in $\mathrm{TOP}^{+}$, top1, top2, and top1-top2 cells harvested either at $25^{\circ} \mathrm{C}$, or after $1 \mathrm{hr}$ of culture at $37^{\circ} \mathrm{C}$. To measure transcription, the steady-state abundance of precursor RNAs was estimated by an S1 nuclease protection assay by use of either of the two probes shown in the diagram $(t o p)$ [the EcoRI (E) and HindIII (H) sites on either side of the enhancer are included for reference]. The 5 '-end probe overlapped the start site of transcription. The ITS 1 probe protects a region of the first internal transcribed spacer that is $2.6 \mathrm{~kb}$ downstream of the start site. (A) Transcription probed at the $5^{\prime}$ end of the large precursor RNA. The abundance of RNA from the $5^{\prime}$ end of the primary transcript is essentially identical in the four strains. Neither the absence of topoisomerase I in top1 cells nor the inactivation of topoisomerase II in top 2 cells cultured at $37^{\circ} \mathrm{C}$ has any significant effect on initiation by RNA polymerase I. Inactivating both topoisomerases by culturing the top1-top2 cells at $37^{\circ} \mathrm{C}$ is also without effect. Therefore, initiation on the endogenous ribosomal genes can proceed in the absence of both topoisomerases. $(B)$ Transcription probed $2.6 \mathrm{~kb}$ downstream from the start site. The level of transcription through ITS 1 is not altered by disruption of topoisomerase I in top1 cells, or the loss of topoisomerase II in top 2 cells cultured at $37^{\circ} \mathrm{C}$. However, in the double mutant at $37^{\circ} \mathrm{C}$, few polymerases transcribe through ITS 1 . From this it is clear that topoisomerase activity is required for chain elongation.

when closed circular plasmids were used as template. This effect is shown in Figure 6. Linear templates also show increased transcription in the mutant extract, but this increase is typically lower, in the range of twofold. The preferential transcription of circular plasmids in the mutant extracts encouraged us to believe that RNA polymerase I might be responding to the superhelical state of the template and led to the experiments shown in Figures 7 and 8 .
Negative supercoils persist in plasmids incubated in extracts

from topoisomerase mutant strains

It is well known that under normal conditions of culture, deproteinized plasmids isolated from bacteria are negatively supercoiled and therefore migrate faster upon standard agarose gel electrophoresis than do linear or relaxed molecules of the same size. In Figure 7 we show that these negative supercoils are eliminated rapidly in an extract from wild-type yeast but persist to varying degrees in extracts made from strains with mutant topoisomerases. In the wild-type $\left(\mathrm{TOP}^{+}\right)$extract, plasmids are relaxed to their equilibrium distribution of supercoils in $<7.5 \mathrm{~min}$ of incubation. In the top1-top2 double mutant extract, negative supercoils persist for at least $15 \mathrm{~min}$. Persistence is observed somewhat less in the top1 single mutant and is not detectable in the top 2 single mutant.

Initiation by RNA polymerase I is stimulated by negative superhelicity

Because negative supercoils can persist in extracts from the double mutant, we were able to examine the ability of RNA polymerase I to initiate transcription on templates with varying degrees of negative superhelicity. Aliquots of a closed circular template were incubated in the presence of topoisomerase I and various amounts of the DNA intercalator, ethidium bromide. After each al-

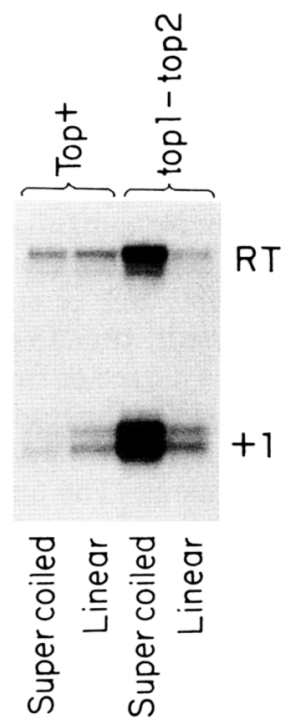

Figure 6. Initiation by RNA polymerase I in whole-cell extracts from $\mathrm{TOP}^{+}$and top1-top2 strains. Templates carrying the ribosomal gene promoter, either linear or negatively supercoiled, were transcribed in $100,000 \mathrm{~g}$ supernatants from extracts of $\mathrm{TOP}^{+}$or top1-top2 cells grown at $25^{\circ} \mathrm{C}$. Transcription was monitored by S1 nuclease protection. Correctly initated polymerase I transcripts protect a 35-nucleotide fragment of the probe $(+1)$; transcripts coming from upstream readthrough $(\mathrm{RT})$ protect the entire 50-nucleotide probe. Supercoiled templates are disproportionately active in the top1-top2 extract. 


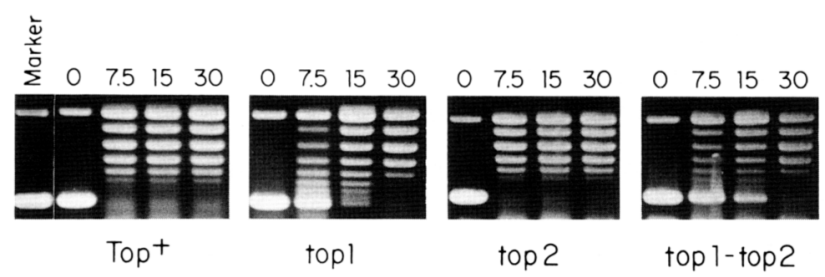

Figure 7. The effect of extracts from topoisomerase mutants on the supercoiling of circular templates. The topology of supercoiled templates was analyzed during transcription in $100,000 \mathrm{~g}$ supernatants from extracts of $\mathrm{TOP}^{+}$, top1, top2, and top1-top2 cells grown at $25^{\circ} \mathrm{C}$. Reactions were stopped at $0,7.5$, 15 , and $30 \mathrm{~min}$, and the templates were isolated and resolved on agarose gels. DNA was stained with ethidium bromide. Most of the added template carried a very high density of negative supercoiling (bottom band in marker lane), although some relaxed, closed circular molecules were also present /top band in marker lane). In the $\mathrm{TOP}^{+}$extract, all of the template is relaxed to the equilibrium distribution of supercoiling in $<7.5 \mathrm{~min}$. The same effect is observed in the top 2 extract. In the top 1 extract, the high negative supercoiling of some templates is retained for at least $15 \mathrm{~min}$. An even greater proportion of the templates persists as highly supercoiled molecules in the top 1-top2 extract. By comparing the data here with the transcription signals in Fig. 6 , it is clear that initiation activity in the extract parallels the persistence of negative supercoiling.

iquot had reached equilibrium, it was deproteinized and the ethidium bromide was removed. As shown in Figure 8 (top), this resulted in a series of templates with different degrees of superhelicity ranging from relaxed to highly negatively supercoiled. Each of these templates was then incubated in an extract from the top1-top2 double mutant strain for 15 min under transcription conditions. (Before the extract was made, the cells were grown at $37^{\circ} \mathrm{C}$ to ensure inactivation of topoisomerase II.) At the end of the reaction, an aliquot was deproteinized and the amount of superhelicity remaining was assessed, as shown in Figure 8 (middle). Extracts made from the double mutant are remarkably free of either nicking or relaxing activities. As a result, after $15 \mathrm{~min}$, the templates retained nearly the same degree of superhelicity as they had when they were added to the reaction. Figure 8 (bottom/ shows the transcription initiation signal resulting from each of the templates. The initiation signal increases directly as the degree of supercoiling in the template increases. In this particular experiment, the initiation signals varied over a fivefold range, from lowest (lane 1, most relaxed) to highest (lane 5, most negatively supercoiled). We conclude that initiation by yeast RNA polymerase I is strongly stimulated by negative superhelicity.

\section{Discussion}

The results reported above provide several new insights into the role of topoisomerases in RNA polymerase I transcription in yeast. First, the inactivation of topoisomerases can lead to a paradoxical stimulation of the initiation of transcription, both in vivo and in vitro. We provide evidence for at least one mechanism that can account for this stimulation. We also provide additional evidence for the requirement of a topoisomerase for efficient RNA chain elongation in vivo.

Once we understand that inactivation of topoisomerase can have both positive and negative effects on RNA polymerase I transcription, we can suggest plausible explanations for many of the in vivo observations presented in Figures 2-5. Consider, first, the transcription of ribosomal genes on extrachromosomal plasmids (Fig. 2). The general result is that inactivation of topoisomerases causes an increase in transcription. A major reason for this increase could be increased initiation owing to the accumulation of negative superhelicity. We have shown (Fig. 8) that initiation by polymerase $I$ is stimulated in vitro by negative supercoiling, and an increase in negative supercoiling was observed in vivo on transcriptionally active extrachromosomal plasmids in top1 and top1-top2 mutants (Brill and Sternglanz 1988). Theoretical considerations also suggest that topoisomerase inactivation should cause at least a transient
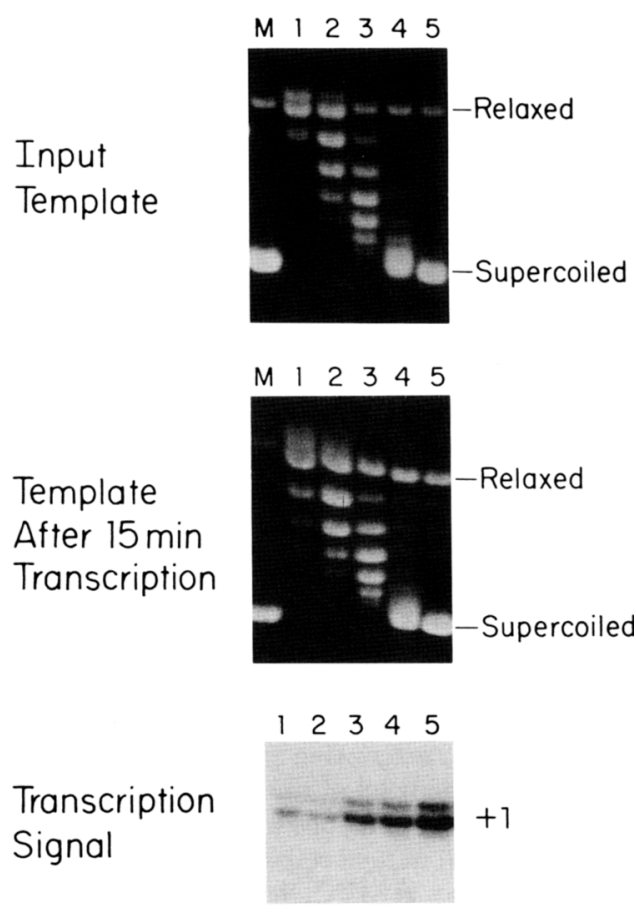

Figure 8. Initiation by RNA polymerase I is stimulated by negative superhelicity. Templates that ranged in their level of negative supercoiling from fully relaxed (lane 1 of agarose gel in top) to highly supercoiled (lane 5, top) were transcribed for $15 \mathrm{~min}$ in the $100,000 \mathrm{~g}$ supernatant from top1-top2 cells grown at $25^{\circ} \mathrm{C}$ and then cultured for $1 \mathrm{hr}$ at $37^{\circ} \mathrm{C}$. Analysis of the templates at the end of the reaction revealed that their relative levels of negative supercoiling did not change during transcription (middle). Initiation from these templates (lanes 1-5, bottom) was monitored by S1 nuclease protection. Evidence that the initiation detected here is the result of RNA polymerase I is given in two previous papers (Schultz et al. 1991; Choe et al. 1992). The level of initiation clearly increases as the density of negative supercoiling of the templates increases. 
buildup of negative superhelicity in the vicinity of the promoter (Liu and Wang 1987).

When the same transcription units are inserted into a chromosome (at the URA3 locus; Fig. 3), the response to topoisomerase inactivation changes. On a short transcription unit, where transcription is being measured near the initiation site, transcription still increases in the double mutant (Fig. 3A). This may indicate that topoisomerase inactivation can cause negative superhelicity to accumulate within the chromosome as well as on an extrachromosomal plasmid. In contrast, when the accumulation of a long transcription product is measured (Fig. 3B), transcription decreases in the double mutant. This suggests that positive superhelicity induced by the elongation process is not able to diffuse away in a chromosomal location and production of long transcripts is inhibited even though initiation has been stimulated.

Figure 4 indicates that essentially the same response is seen when constructs are integrated into the ribosomal gene locus instead of the URA3 locus. Transcription of short constructs is stimulated in the double mutant (Fig. $4 \mathrm{~A}, \mathrm{~B})$ while the ability to produce long transcripts is impaired (Fig. 4C,D). The stimulation of short transcripts appears to be less than that observed at the URA3 locus, suggesting that the accumulation of negative superhelicity in the chromosome may vary quantitatively from one locus to another.

The observations made on the endogenous ribosomal genes (Fig. 5) can also be harmonized with the above interpretation. When measurements of transcription are made at the extreme $5^{\prime}$ end of the transcription unit, no change in signal is seen upon topoisomerase inactivation (Fig. 5A). This is the most direct evidence available that topoisomerase is not required for normal rates of polymerase I initiation. It suggests further that in contrast to extrachromosomal plasmids, significant negative superhelicity does not accumulate on the endogenous ribosomal genes in the double mutant. When transcription is measured farther down the endogenous transcription unit (Fig. 5B), a severe inhibition is observed in the double mutant. This agrees with the previous observation that accumulation of mature rRNA is inhibited in the double mutant and strongly supports the conclusion that a failure of chain elongation is the cause of this inhibition.

A related study has been reported by Zhang et al. (1988), who observed a severe decrease in rRNA synthesis in human cells after treatment with camptothecin. Interpretation of this result was made difficult by the fact that camptothecin inhibits topoisomerase I by trapping it as a covalent adduct with the DNA. Thus, the decrease in chain elongation could have been the result of either a lack of topoisomerase activity or physical blockage of the polymerase by protein linked to the DNA template.

The study of Zhang et al. (1988) did provide direct evidence that topoisomerase I is localized on the rDNA transcription unit. This agrees with a similar study that also used camptothecin to trap topoisomerase I in situ (Culotta and Sollner-Webb 1988). Previous reports have also arrived at the same conclusion by use of different techniques. For example, Higashinakagawa et al. (1977) purified amplified oocyte nucleoli away from detectable chromosomal DNA and showed that the nucleoli that were most active in transcription also had the highest amount of topoisomerase I activity. Antibodies against topoisomerase I also reveal that the protein is localized to actively transcribing genes, among which the nucleolus is highly prominent (Fleischman et al. 1984; Muller et al. 1985). Thus, the physical location of topoisomerase I is consistent with its apparent role in promoting RNA chain elongation.

Although we can rationalize some of the effects of mutating topoisomerases, we must emphasize that there are still aspects of the in vivo response that are difficult to understand. This especially applies to the effects of mutating topoisomerase II. The studies of Brill et al. (1987) and Yamagishi and Nomura (1988), as well as the work reported in this paper, show that topoisomerase II can substitute for topoisomerase I in promoting chain elongation. This suggests that significant topoisomerase II must be present in the nucleolus even though localization studies with immunoflourescent antibodies show it to be localized to the chromosomal scaffold (Earnshaw et al. 1985) and not particularly enriched in the nucleolus. We are especially puzzled by the fact that, in several instances, inactivation of topoisomerase II alone causes a dramatic increase in the transcription signal (see Figs. 2 and 3). Inactivation of topoisomerase I, by itself, also causes an increase in transcription, but this is readily explained, as topoisomerase I appears to be the most abundant relaxing activity in the cell. In extracts made from the top1 mutant strain, negative supercoils persist longer than in wild-type extracts (Fig. 7). In contrast, in extracts made from the top2 mutant strain, an abundance of topoisomerase I still remains and negative supercoils are rapidly relaxed (Fig. 7). Thus, it is difficult to explain the increase in transcription seen in top2 strains by invoking an accumulation of negative superhelicity. [Brill and Sternglanz (1988) also did not observe an accumulation of negative superhelicity in top 2 strains in vivo.] These findings suggest some as yet unexpected role of topoisomerase II in ribosomal gene transcription.

\section{Materials and methods}

Strains

Four closely related strains of $S$. cerevisiae were used for the in vitro experiments and were also transformed with artificial rDNA constructs as described below: a wild-type strain, W3031a: MATa ade2-1 trp1-1 can1-100 leu2-3,112 his3-11,15 ura3-1), originally from R. Rothstein (Columbia University, New York); RS190 (W303-1a top1-8::LEU2); RS191 (MATa ade2-1 trp1-1 can1-100 leu2-3,112 his3-11,15 ura3-1 top2-1); and RS192 (RS191 top1-8::LEU2). The production of these strains is described in Brill and Sternglanz (1988).

In vivo analysis of transcription from artificial rRNA genes

W303-1a, RS190, RS191, and RS192 were transformed with a series of rDNA constructs. 
Constructs on plasmids rDNA constructions were cloned into the centromere plasmid YCp50 (Rose et al. 1987; Fig. 1A). The parent construct rR8 (Elion and Warner 1984) contains the 2.35$\mathrm{kb}$ HindIII-BgIII fragment, which includes $\sim 120$ bp of the external transcribed spacer, the $35 \mathrm{~S}$ gene promoter, and $2 \mathrm{~kb}$ of upstream spacer sequences. The coding sequence is followed by a unique 600 -bp fragment of phage T7 DNA that allows for detection of transcripts from the polymerase I promoter of this minigene as a $1.2-\mathrm{kb}$ product. The $\mathrm{T} 7$ insert is followed by a 590-bp EcoRI fragment spanning the $3^{\prime}$ end of the coding region. YCprR10 (Elion and Warner 1984) is the same as YCprR8, except that the 190-bp EcoRI-HindIII fragment of the enhancer has been restored to the 5' end of the 2-kb spacer segment. The maxigene YCprR6.1 was made from YCprR8 by enlarging the insert of unique marker DNA; a 4.9-kb EcoRI fragment of $\lambda$ phage DNA was cloned into the EcoRI site downstream of the 1.2 -kb segment that includes rDNA-coding sequences and the T7 DNA. The rR6.2 maxigene is the same as rR6.1, except that the 190-bp enhancer fragment has been restored to the $5^{\prime}$ end of the $2-\mathrm{kb}$ spacer segment. The $6.1-\mathrm{kb}$ product of the rR6 maxigenes is about the same length as the primary transcript of the $35 \mathrm{~S}$ gene. These plasmids were maintained in host strains grown on the appropriate selective minimal medium.

Integrated constructs $\mathrm{rR} 10$ and $\mathrm{RR} 6.1$ (Fig. 1B, diagrams 5,6) in the YIp5 vector (Struhl et al. 1979) were integrated into the ura3-1 locus of W303-1a, RS190, RS191, and RS192 by standard techniques (see Elion and Warner 1984). W303-1a was similarly transformed with rR6.1 (to give RS320), but this insertion was obtained only rarely. Therefore, the construct was moved to other backgrounds by genetic means. The isogenic strain of opposite mating type was obtained by crossing RS320 with the isogenic strain W303-1b (MAT $)$. A URA3 segregant was then crossed to RS192 to yield top1, top2, and top1-top2 segregants. rR8 and rR6.1 were either integrated (Fig. 1B, diagrams 1,3) or transplaced (Fig. 1B, diagrams 2,4) into the rDNA repeat of hosts with variously mutated topoisomerases. $\mathrm{rR} 8$ was integrated into the repeat of strains RS188, RS190, RS191, and RS192 by use of YIprR3 as described in Elion and Warner (1984). rR6.1 was integrated into the repeat by use of YIprR3L, which is YIprR3 expanded with the 4.9-kb EcoRI fragment of $\lambda$ phage DNA described above. TOP ${ }^{+}$and top1-top2 strains with rR6.1 integrated into the repeat were obtained by transformation of W3031a and RS192, respectively, with gel-purified linear YIprR3L cut partially with HindIII. Integration places rR8 and rR6.1 between an endogenous enhancer and the URA3 gene. Strains with rR8 transplaced were obtained by cotransformation of W303-1a and RS192 with $50 \mathrm{ng}$ of circular YEp24 DNA and $100 \mathrm{ng}$ of pHB3.5 (3.5-kb HindIII-BamHI fragment of YIprR3 in pBR322) DNA cut completely with HindIII and BamHI. YEp24 plasmid was cured from the strains before use. This construction could not be maintained by selective pressure and was lost frequently. In addition, it was obtained rarely and could not be found in segregants of genetic crosses. W303-1a and RS192 with rR6.1 transplaced to the rDNA repeat (to give RS281 and RS293, respectively) were generated by cotransformation with $50 \mathrm{ng}$ of circular YEp24 plasmid DNA and >100 ng of a gel-purified 8.1-kb fragment of YIprR3L DNA obtained after partial digestion with HindIII and complete digestion with StuI. This construction was maintained stably in crosses; therefore, top1 and top2 strains could be obtained from a cross of RS293 to W303-1b. $U R A 3$ transformants were screened by Southern blotting to confirm the orientations shown in Figure 1B.

To measure transcription from the introduced genes, cells were grown in YEPD at $25^{\circ} \mathrm{C}$ to an $A_{600}$ of 1 . The culture was split, and total nucleic acids were prepared immediately from one aliquot, according to Brill and Sternglanz (1988). Nucleic acids were also prepared from cells that were cultured further at the nonpermissive temperature, $37^{\circ} \mathrm{C}$, for $1-3 \mathrm{hr}$. Transcription of rRNA minigenes was monitored by Northern blotting as described in Brill and Sternglanz (1988), with ${ }^{32}$ P-labeled T7 DNA (Elion and Warner 1984). As internal controls the abundance of transcripts from either the CYR1 gene or the TY1 gene was measured on the same blot by use of appropriate probes.

\section{In vivo analysis of transcription}

from the endogenous $35 S \mathrm{rRNA}$ genes

Strains W303-1a, RS190, RS191, and RS192 were grown in $50 \mathrm{ml}$ of YEPD at $25^{\circ} \mathrm{C}$ to an $A_{600}$ of 2 . Each culture was split in two, and total nucleic acids were prepared immediately from one aliquot while the remaining cells were cultured at $37^{\circ} \mathrm{C}$ for $1 \mathrm{hr}$ before the isolation of nucleic acids. For this analysis, sodium azide was added to the cultures immediately before breaking the cells. Transcription was measured by steady-state analysis of precursor RNAs using an S1 nuclease protection assay and endlabeled single-stranded DNA probes. Probe $\left(2.5 \times 10^{5} \mathrm{cpm}\right)$ was hybridized to $2 \mu \mathrm{g}$ of total nucleic acids. A 40 -mer oligonucleotide (probe $5^{\prime}$ end; Fig. 5), complementary to the region of the primary transcript from -10 to +30 , was used to detect the very $5^{\prime}$ end of the $35 \mathrm{~S}$ precursor. Hybridization with this probe was performed at $50^{\circ} \mathrm{C}$. A 40 -mer oligonucleotide (probe ITS 1; Fig. 5), complementary to the region of ITS 1 from 419 to 458 following the numbering of Veldman et al. (1980), was used to detect primary transcripts of $2.6 \mathrm{~kb}$ in length. This region of the primary transcript includes the early processing site A2 (Klootwijk and Planta 1989). Full-length protection of this probe, therefore, is due to unprocessed $35 \mathrm{~S}$ precursor, as well as molecules that have been cleaved at site $\mathrm{Al}$, but not at $\mathrm{A} 2$. Because the region detected by this probe is not present in mature rRNAs, there is no background in this assay owing to the stored pool of cytoplasmic ribosomes. Probe ITS 1 was hybridized to nucleic acid samples at $45^{\circ} \mathrm{C}$.

In vitro analysis of rDNA transcription

in extracts from strains with mutated topoisomerases

W303-la, RS190, RS191, and RS192 cells were grown at $25^{\circ} \mathrm{C}$ to an $A_{600}$ of 1 . Half of the cells were harvested immediately; the other half were cultured at $37^{\circ} \mathrm{C}$ for $\mathrm{l} \mathrm{hr}$ and then harvested. Transcription extracts were prepared as $100,000 \mathrm{~g}$ supernatants, according to Schultz et al. (1991), by use of a mortar and pestle to break the cells. The protein content of the extracts varied between 17 and $22.4 \mathrm{mg} / \mathrm{ml}$. Transcription reactions were performed as described [Schultz et al. 1991), except that they included $90 \mathrm{mM} \mathrm{KCl}$, and the NTPs were at $500 \mu \mathrm{m}$ each. The reactions contained $2.1 \mathrm{mg} / \mathrm{ml}$ of extract protein and $30 \mu \mathrm{g} / \mathrm{ml}$ of template in a total volume of $20 \mu \mathrm{l}$ and were run for $15 \mathrm{~min}$ at $25^{\circ} \mathrm{C}$. pYrl1 A (Schultz et al. 1991) was the template for all reactions. This plasmid contains a 220 -bp fragment that includes the entire polymerase I promoter. A linker inserted at +25 allows for detection of only those polymerase I transcripts arising from the added template. Initiation was monitored with an end-labeled 50-mer oligonucleotide in an S1 nuclease protection assay (Schultz et al. 1991). This probe spanned the region of pYr11A from -15 to +35 . One-fifth of each reaction was analyzed with $2.5 \times 10^{5} \mathrm{cpm}$ of probe. Templates with varying densities of negative superhelicity were prepared according to Singleton and Wells (1982); pYr11A was cut with BglI when used as a linear. 


\section{Template topology assay}

Templates were purified from in vitro transcription reactions by extraction with phenol and precipitating total nucleic acids as for the in vitro analysis of RNA synthesis. The topology of templates was monitored by electrophoresis in $0.8 \%$ agarose/ TAE ( $40 \mathrm{~mm}$ Tris-acetate, $2 \mathrm{~mm}$ EDTA) or $0.7 \%$ agarose/TBE $(89 \mathrm{~mm}$ Tris-borate, $89 \mathrm{~mm}$ boric acid, $10 \mathrm{~mm}$ EDTA). DNA was visualized by staining with ethidium bromide after running the gel.

\section{Acknowledgments}

We thank Jon Warner for valuable advice in the early stages of this work and are grateful to him and Elaine Elion for generously supplying several plasmids and probes. This work was supported by National Institutes of Health grants GM28220 to R.S., GM26624 to R.H.R., and GM25532 to Jon Warner.

The publication costs of this article were defrayed in part by payment of page charges. This article must therefore be hereby marked "advertisement" in accordance with 18 USC section 1734 solely to indicate this fact.

\section{References}

Brill, S.J. and R. Sternglanz. 1988. Transcription-dependent DNA supercoiling in yeast DNA topoisomerase mutants. Cell 54: 403-411.

Brill, S.J., S. DiNardo, K. Voelkel-Meiman, and R. Sternglanz. 1987. Need for DNA topoisomerase activity as a swivel for DNA replication and for transcription of ribosomal RNA. Nature 326: 414-416.

Choe, S.Y., M.C. Schultz, and R.H. Reeder. 1992. In vitro definition of the yeast RNA polymerase I promoter. Nucleic Acids Res. 20: 279-285.

Culotta, V. and B. Sollner-Webb. 1988. Sites of topoisomerase I action on $\mathrm{X}$. laevis ribosomal chromatin: Transcriptionally active rDNA has an $\sim 200$ bp repeating structure. Cell 52: 585-597.

Dunaway, M. 1990. Inhibition of topoisomerase II does not inhibit transcription of RNA polymerase I and II genes. Mol. Cell. Biol. 10: 2893-2900.

Earnshaw, W.C., B. Halligan, C.A. Cooke, M.M.S. Heck, and L.F. Liu. 1985. Topoisomerase II is a structural component of mitotic chromosome scaffolds. I. Cell Biol. 100: 1706-1715.

Elion, E.A. and J.R. Warner. 1984. The major promoter element of rRNA transcription in yeast lies $2 \mathrm{~kb}$ upstream. Cell 39: 663-673.

Fleischman, G., G. Pflugfelder, E. Steiner, K. Javaherian, G. Howard, J. Wang, and S. Elgin. 1984. Drosophila DNA topoisomerase $\mathrm{I}$ is associated with transcriptionally active regions of the genome. Proc. Nat1. Acad. Sci. 81: 6958-6962.

Giaever, G.N. and J.C. Wang. 1988. Supercoiling of intracellular DNA can occur in eukaryotic cells. Cell 55: 849-856.

Higashinakagawa, T., H. Wahn, and R.H. Reeder. 1977. Isolation of ribosomal gene chromatin. Dev. Biol. 55: 375-386.

Klootwijk, J. and R.J. Planta. 1989. Isolation and characterization of yeast ribosomal RNA precursors and preribosomes. Methods Enzymol. 180: 96-109.

Liu, L.F. and J.C. Wang. 1987. Supercoiling of the DNA template during transcription. Proc. Natl. Acad. Sci. 84: 7024-7027.

Muller, M., W. Pfund, V. Mehta, and D. Trask. 1985. Eukaryotic type I topoisomerase is enriched in the nucleolus and catalytically active on ribosomal DNA. EMBO \%. 4: 1237-1243.

Rose, M.D., P. Novick, J.H. Thomas, D. Botstein, and G.R. Fink.
1987. A Saccharomyces cerevisiae genomic plasmid bank based on a centromere-containing shuttle vector. Gene 60: $237-243$.

Schultz, M.C., S.Y. Choe, and R.H. Reeder. 1991. Specific initiation by RNA polymerase I in a whole-cell extract from yeast. Proc. Natl. Acad. Sci. 88: 1004-1008.

Singleton, C.K. and R.D. Wells. 1982. The facile generation of covalently closed, circular DNAs with defined negative superhelical densities. Anal. Biochem. 122: 253-257.

Struhl, K., D.T. Stinchcomb, S. Scherer, and R.W. Davis. 1979. High frequency transformation of yeast: Autonomous replication of hybrid DNA molecules. Proc. Nat1. Acad. Sci. 76: $1035-1039$.

Veldman, G.M., R.C. Brand, J. Klootwijk, and R.J. Planta. 1980. Some characteristics of processing sites in ribosomal precursor RNA of yeast. Nucleic Acids Res. 8: 2907-2920.

Wu, H.-Y., S. Shyy, J.C. Wang, and L. Liu. 1988. Transcription generates positively and negatively supercoiled domains in the template. Cell 53: 433-440.

Yamagishi, M. and M. Nomura. 1988. Deficiency in both type I and type II DNA topoisomerase activities differentially affect rRNA and ribosomal protein synthesis in Schizosaccharomyces pombe. Curr. Genet. 13: 305-314.

Zhang, H., J.C. Wang, and L.F. Liu. 1988. Involvement of DNA topoisomerase I in transcription of human ribosomal RNA genes. Proc. Natl. Acad. Sci. 85: 1060-1064. 


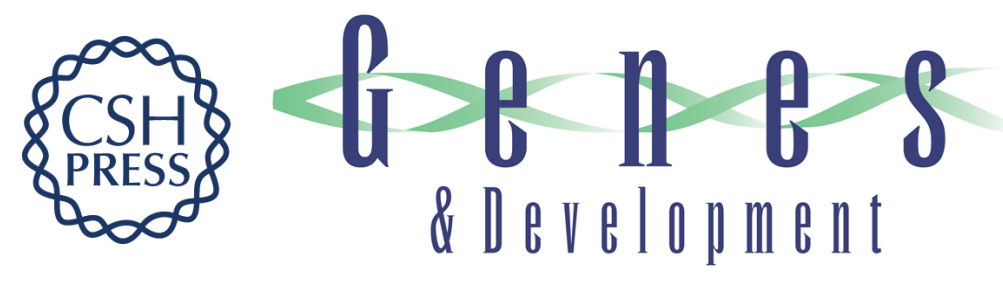

\section{Topoisomerases and yeast rRNA transcription: negative supercoiling stimulates initiation and topoisomerase activity is required for elongation.}

M C Schultz, S J Brill, Q Ju, et al.

Genes Dev. 1992, 6:

Access the most recent version at doi:10.1101/gad.6.7.1332

References This article cites 21 articles, 7 of which can be accessed free at:

http://genesdev.cshlp.org/content/6/7/1332.full.html\#ref-list-1

License

Email Alerting

Service

Receive free email alerts when new articles cite this article - sign up in the box at the top right corner of the article or click here.

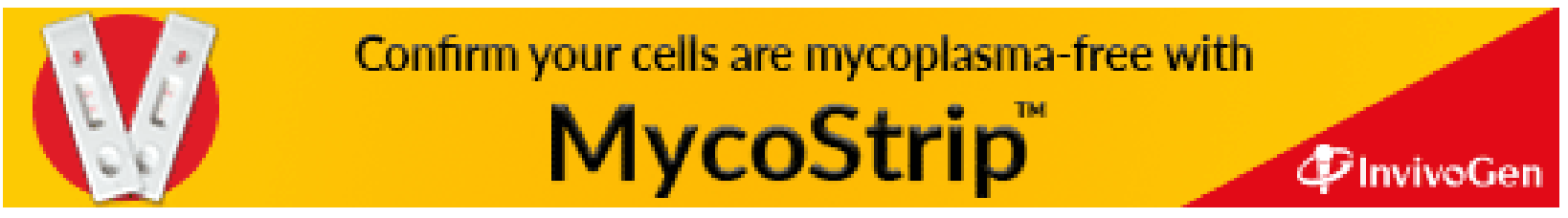

